\title{
Epigenetic ecotypes in animals: persistent environmental adaptation in the absence of genetic variation
}

\author{
Günter $\operatorname{Vogt}^{1}$ \\ ${ }^{1}$ University of Heidelberg Faculty of Biosciences
}

March 2, 2022

\begin{abstract}
According to prevailing theory, sexually reproducing animals adapt to different environments by the production of phenotypic variation from the standing genetic variation and selection of the most suited phenotypes. Contrary to all expectations, asexually reproducing animals can also inhabit broad ranges of geographical latitudes, altitudes and habitats, despite virtual genetic identity. Recent whole genome analyses of differently adapted clonal populations and genetically impoverished invaders revealed that they can use epigenetic variation instead of genetic variation to stably adapt to different environments. The required phenotypes are produced from the same DNA sequence via changes in gene expression, which is trigged by strong environmental cues and mediated by environment-sensitive epigenetic mechanisms like DNA methylation. Habitat-specific epigenetic fingerprints were maintained over subsequent years, pointing at the existence of epigenetic ecotypes. Obviously, all animals can produce different phenotypes from the same DNA sequence, but in asexually reproducing populations, genetically impoverished invaders, sessile taxa and species with long generation times it is apparently of prime importance. In contrast to beneficial genetic mutations and meiotic gene combinations that require many generations to be established in a population, environmentally-induced epigenetic changes and subsequent alterations in gene and phenotype expression affect population members synchronously in the first exposed generation, providing an ideal means for fast, directional adaptation to changing conditions. The production of different phenotypes from the same genome in response to different environmental cues via epigenetic mechanisms is also suitable to explain the "general-purpose genotype" and the "genetic paradox of invasion".
\end{abstract}

\section{Introduction}

The adaptation of organisms to different environments requires phenotypic variation on which natural selection can act. In sexually reproducing organisms, phenotypic variation is produced from the standing genetic variation, and different selective forces in different environments then trigger the evolution of ecotypes ( Lowry, 2012; Malec et al., 2021). Interestingly, asexually reproducing organisms can also inhabit very broad ranges of geographical regions and habitats and stably adapt to them despite the scarcity or virtual absence of genetic variation (Peck et al., 1998; Mackay-Smith et al., 2021), which is often explained by the existence of so-called "general-purpose genotypes" (Massicotte \& Angers, 2012). The same holds for small invasive groups that can be very successful in new environments never experienced before despite paucity of genetic variation, which is known as the "genetic paradox of invasion" (Sax \& Brown, 2000, Estoup et al., 2016).

Recent work with clonal organisms and genetically impoverished invasive groups revealed that epigenetic mechanisms can change expression of the same DNA sequence, either stochastically or in response to environmental signals, leading to different phenotypes (Verhoeven \& Preite, 2014; Vogt, 2017, 2020a, 2021; Angers et al., 2020). Therefore, epigenetically-based phenotypes are prime candidates for the explanation of the "general-purpose genotype" and the "genetic paradox of invasion". Epigenetic mechanisms include DNA methylation, histone modifications and non-coding RNAs (Jaenisch \& Bird, 2003; Lennartsson \& Ekwall, 2009; Moutinho \& Esteller, 2017). Monoclonal organisms are particularly suitable to investigate environ- 
mental adaptation by epigenetic mechanisms, because genetic variation is absent or very small in these test systems and does not camouflage the phenotypic variation produced by epigenetic mechanisms.

Previously, the analysis of epigenetic patterns on the whole genome scale in statistically relevant numbers of individuals was hampered by very high costs, the limited sensitivity of the methods and the need of large amounts of tissue per measurement, often requiring pooling of specimens. The recent development of affordable, highly sensitive and relatively fast genetic and epigenetic approaches like third generation sequencing (Van Dijk et al., 2018) and whole genome bisulfite sequencing (Kernaleguen et al., 2018) makes it now possible to investigate these issues in depth.

The term epigenetic phenotypes is used in the following for phenotypes that originate from the same DNA sequence by differential gene expression. These phenotypes share the same genes but differ in epigenetic marks and phenotypic traits. Epigenetic variation is the variation of epigenetic marks and resulting phenotypes in a population. The term epigenetic ecotypes describes groups of organisms within asexually reproducing or genetically impoverished species that are stably adapted to different environments. Such groups are genetically identical or very similar among each other, but differ morphologically, physiologically and behaviourally and show persistent group-specific epigenetic signatures.

\section{Generation of multiple phenotypes from the same genotype by epigenetic mechanisms}

The first question to be discussed is how the same genome can produce multiple morphological, physiological, behavioural and life-history phenotypes. In animals, this phenomenon is most easily recognizable by the production of structurally and functionally diverse cell types from the DNA of the zygote (Vickaryous \& Hall, 2006) and the morphologically and behaviourally different life stages and castes of holometabolous insects (Simpson et al., 2011). Less obvious is the generation of different phenotypes from the same genome within populations. The underlying mechanism is differential gene expression with the help of regulatory epigenetic mechanisms. DNA methylation, histone modifications and non-coding RNAs can modify the accessibility of the chromatin and DNA for sequence-reading and interpreting molecules and help in processing of the transcripts.

The contribution of epigenetic mechanisms to phenotypic variation is best studied in populations with zero or low genetic variation. This is achieved by performing experiments with monozygotic twins and polyembryonic multiples of mammals (Ballouz et al., 2019; Van Dongen et al., 2021), clonal fish (Leung et al., 2016), genets of coral colonies (Liew et al., 2018) and clone-mates of apomictic parthenogenetic invertebrates such as Daphnia water fleas (Kvist et al., 2020), Artemia brine shrimps (Norouzitallab et al., 2016), snailPotamopyrgus antipodarum (Thorson et al., 2017) and crayfishProcambarus virginalis (Vogt, 2022).

Epigenetic variation can result from stochastic or environmentally induced epimutations (Feinberg \& Irizarry, 2010; Verhoeven \& Preite 2014; Angers et al., 2020; Vogt, 2020a, 2021). Epimutations are principally reversible changes in the chemical structure of the DNA that, unlike genetic mutations, do not change the DNA sequence. In the model plant Arabidopsis thaliana, epimutations are about five orders of magnitude more frequent than genetic mutations $\left(10^{-4}\right.$ versus $10^{-9}$ per base pair and generation) (Van der Graaf et al., 2015). In wild populations, both sources of epigenetic variation occur together but in different weighing, depending on conditions. In clonal fish Chrosomus eos-neogaeus, directional environment-induced epigenetic variation was predominant in predictable environments, but bet-hedging stochastic epigenetic variation prevailed in unpredictable environments, suggesting that both strategies were differentially selected (Leung et al. 2016).

The environmental induction of epigenetic variation via differential gene expression, which is at the centre of this paper, is triggered by strong environmental cues like temperature, salinity, food, predator odours, toxicants or disease agents (Skinner, 2014; Guillette et al., 2016). It requires signal transmission from the external world to the nucleus of the target cells, environment-sensitive molecules involved in gene regulation, readers and editors of epigenetic marks, and molecules that recruit the epigenetic modifiers to specific regions of the DNA and chromatin (Figure 1). 
Some environmental cues can directly act on the target cells, e.g., fatty acids from the food, but most of them are first perceived by sense organs and translated into neurohormonal signals that are then conveyed to the target cells. The hormonal signals elicit cellular signals like second messengers, which finally regulate molecules involved in chromatin remodelling, gene expression and processing of the transcripts. Serotonin is a good example of an environmental signal transmitting hormone. In locust polyphenism, the density-dependent change of morphologically and behaviourally different stationary and migratory phases, it contributes to the alternative expression of density-sensitive genes with the help of epigenetic mechanisms (Foquet et al., 2021).

A considerable number of the enzymes participating in regulation of chromatin architecture and gene expression are apparently responsive to environmental cues. Examples are the DNA demethylating ten-eleven translocation methylcytosine dioxygenase (TET), which is up- or downregulated by several environmental factors including food ingredients, ethanol, air pollution and radiation (Zhu et al., 2020), proteins of the Polycomb group that are sensitive to the environmental temperature (Voigt \& Kost, 2021), and transcription factors of the TCP family in plants that mediate environmental signals into growth responses (Danisman, 2016). In order to change gene expression, environment-sensitive proteins that are able to modify the epigenetic signatures of the chromatin and DNA must crosstalk with readers of the epigenetic marks and molecules that recruit them to specific sites of the DNA and chromatin (Figure 1).

The writers and erasers of the DNA methylation marks include the methylating DNA methyltransferases (DNMTs) and the demethylating TET (Wu \& Zhang, 2017; Lyko, 2018). These enzymes form complexes with readers of the DNA methylation marks like proteins of the methyl-CpG-binding domain family (MBDs) and transcription factors to exert their functions (Huang et al., 2018; Ravichandran et al., 2018). The MBDs bind methylated $\mathrm{CpG}$ dinucleotides and act as translators between DNA methylation and histone modifications (Du et al., 2015). Transcription factors with different sequence specificity can guide the methylation modifying enzymes to specific sites of the DNA (Kribelbauer et al., 2020). Each animal possesses hundreds of such transcription factors.

The histones of the nucleosomes influence gene expression by either shielding the DNA or allowing binding of transcription factors to the DNA. The N-terminal tails of the histones carry modifications like acetylation, methylation, phosphorylation or ubiquitination, which affect the chromatin structure. Histone acetylation often expands the chromatin and stimulates gene expression, whereas histone methylation often represses gene expression, depending on their location in the histones (Allis \& Jenuwein, 2016). The histone modifications are produced by a broad array of enzymes and read by various proteins (Marmorstein \& Zhou, 2014; Morgan \& Shilatifard, 2020). For example, histone acetylation marks are written by histone acetyltransferases (HATs) and read by bromodomain-containing proteins (BrDs).

\section{Environmental adaptation by epigenetic phenotypes: evidence for epigenetic ecotypes}

Laboratory and field work of the last decade with differently adapted clonal animals, genetically impoverished invaders and sessile species suggest that all of them use epigenetic variation for environmental adaptation. In the following, selected case studies are discussed on the example of DNA methylation.

\subsection{Clonal populations}

The monoclonal populations of the New Zealand mud snail,Potamopyrgus antipodarum, in the western USA are a good example of environmental adaptation by epigenetic mechanisms and the establishment of epigenetic ecotypes. These populations originated from a single clone that was introduced some 35 years ago and are genetically largely identical (Dybdahl \& Drown, 2011). Thorson et al. (2017) found habitat specific differences in shell shape, which were correlated with water current speed (Figure 2A). Using methylated DNA immunoprecipitation (MeDIP) and Illumina sequencing of foot pad tissue, the authors also revealed significant genome-wide DNA methylation differences between lakes and rivers (Figure 2B). These data suggest that environmentally induced epigenetic variation underpins adaptive phenotypic diversity that has been established in less than 100 generations, despite genetic identity. 
Thorson et al. (2019) also compared populations from a rural lake (Lake Lyte, Oregon) and two polluted urban lakes (Capitol Lake and Lake Washington, Washington). They measured differences in shell shape and allometric growth (Figure 2C) and identified numerous differentially methylated DNA regions between the three lakes. A relatively high number of DMRs was shared between rural Lake Lyte and Capitol Lake characterized by high water temperature and high levels of phosphorous and faecal bacteria, and between the two urban lakes. However, there were only a few DMRs shared between the rural lake and Lake Washington heavily polluted by heavy metals and organic xenobiotics (Figure 2D). The presence of site-specific differences in DNA methylation between the genetically identical lake populations suggests epigenetic adaptation to different environmental factors.

The marbled crayfish, Procambarus virginalis, is another good example of environmental adaptation by epigenetic phenotypes. This apomictic parthenogenetic all-female crayfish evolved from a single female of the Floridian slough crayfish, Procambarus fallax , by autotriploidization a few decades ago (Vogt et al., 2008, 2015, 2018, 2019; Martin et al., 2010, 2016; Vogt, 2020b; Legrand et al., 2021). It first appeared in the German aquarium trade in 1995 and was distributed from there across the world. Releases in the last 20 years have led to the establishment of populations in tropical to cold-temperate biomes in more than 20 European, African and Asian countries (coordinates and references in Vogt, 2020b and Maiakovska et al., 2021). Marbled crayfish are now found in a broad range of habitats from lowland to mountain areas including rivers, lakes, ponds, rice fields and acidic, thermal and heavily polluted water bodies (Figure 3A).

High quality reference genomes were assembled from short read and paired-end Illumina HiSeq and long read PacBio Single Molecule Real Time data of single individuals, and a genome-wide reference methylome was established from whole-genome bisulfite sequencing on an Illumina HiSeq platform (Gatzmann et al., 2018; Gutekunst et al., 2018, 2021). Comparison of whole genomes of 19 representatives from 15 wild populations in Europe and Madagascar with the reference genome revealed extremely low genetic variation of a total of 16,564 single nucleotide variants (SNVs) in the $3.7 \mathrm{~Gb}$ genome (Figure 3B). The reference genome was obtained from a descendant of my laboratory population, which was founded in 2003 with a single specimen from the oldest known aquarium lineage started in 1995. Since the generation time of marbled crayfish is about 7 months, the various populations examined are separated from the laboratory reference for no more than 40 generations. About $74 \%$ of the SNVs were located in intergenic regions and $4 \%$ were located in coding regions, among them very few non-synonymous variants that change amino acids in proteins (Gutekunst et al., 2018; Maiakovska et al., 2021).

Despite this genetic uniformity, wild marbled crayfish populations showed considerable phenotypic differences to laboratory-raised populations with respect to growth, coloration, spination and body proportions (Vogt et al., 2018). For example, specimens from my laboratory reached maximum body lengths of only $9 \mathrm{~cm}$ and weights of $18 \mathrm{~g}$, whereas their wild relatives in Lake Moosweiher, Germany, grew to $12 \mathrm{~cm}$ and $40 \mathrm{~g}$ (Figure 3C). In predator-free, acidic Lake Murner See, Germany, they even grew to a maximum of $13 \mathrm{~cm}$ and 52 g (Tönges et al., 2021a). The wild specimens in Lake Moosweiher generally had a uniform dark greenishbrown tanning on the dorsal side, whereas the laboratory reared specimens displayed a broad spectrum of greenish-brown, brown, reddish and bluish dorsal colorations (Figure 3C).

The wild specimens had sharp spines on the carapace and chelipeds, whereas laboratory specimens lacked such spines (Figure 3D). Specimens transferred from Lake Moosweiher to the laboratory maintained their spines through several moults until the end of life, but in the F1 progeny the spines were reduced to small knobs resembling members of the laboratory colony, suggesting that spination may be predator-induced. Laboratory specimens also had relatively longer pleons and broader carapaces when compared to wild specimens (Figure $3 \mathrm{E}$ ). The offspring of a specimen that was transferred from Lake Moosweiher to the laboratory and reproduced there several months later had a total length/carapace length ratio similar to the wild population and their mother, but a carapace length/carapace width ratio more similar to the laboratory population (Figure 3E). The transfer experiments suggest partial morphological adaptation to the new environment.

The phenotypic differences between wild and laboratory specimens were associated with differences in DNA methylation (Tönges et al., 2021b), which were particularly prominent in the hepatopancreas (Figures 3F, 
$\mathrm{G}$ ), the main metabolic organ of crayfish (Vogt, 2019). Moreover, specimens reared at $10^{\circ} \mathrm{C}$ instead of $20^{\circ} \mathrm{C}$ for 6 months exhibited significant differences in average methylation of 361 variably methylated genes providing experimental evidence for environmentally induced methylation changes within a single generation in marbled crayfish (Tönges et al., 2021b).

Significant differences were also found in the population structure between five populations from different bio-climatic regions and habitats in Madagascar (Andriantsoa et al., 2019). An example is given in Figure 3H. Comparison of the methylation patterns of 122 variably methylated genes in the hepatopancreas of specimens from pristine Andragnaro River and polluted Ihosy River in Madagascar and from acidic, oligotrophic Lake Singliser See (no fishes present) and eutrophic Lake Reilinger See in Germany identified specific and highly localized DNA methylation signatures for each population (Figure 3I) (Tönges et al., 2021b). These DNA methylation fingerprints remained stable over consecutive years (Figure 3J). Gene ontology analysis of the variably methylated genes revealed a significant enrichment of GTP-binding proteins, which transmit signals from the external world to the cells regulating various cellular processes. Enrichment was also recorded for proteins involved in regulation of transcription and translation, RNA metabolism, response to stress, and immune response. Since SNVs were absent from the differentially methylated genes investigated, the studies provide conclusive evidence for the independence of epigenetic variation from DNA sequence variation and the existence of epigenetic ecotypes (Tönges et al., 2021b).

Mackay-Smith et al. (2021) studied gene expression adaptation to different host plants in parthenogenetic weevils Naupactus cervinus and Naupactus leucoloma. They revealed that gene expression responses are host plant-specific and that elements of that response are maintained in the offspring. The authors emphasized that modulation of gene expression in the absence of other sources of phenotypic adaptation like genetic variation may be important for successful colonization of different hosts. Unfortunately, the epigenetic mechanisms that regulate gene expression in these weevils are not yet investigated.

\subsection{Sexually reproducing invaders and sessile taxa}

Three examples may illustrate the extent and role of epigenetic phenotype production in sexually reproducing animals: an introduced bird in Kenya and Florida, an experimental invasion of Caribbean islands by a lizard, and a sessile coral inside and outside of the Arabian-Persian-Gulf,

The house sparrow, Passer domesticus, was introduced to Kenya 65 years ago and to Florida 150 years ago and since has evolved significant phenotypic variation in both populations (Schrey et al., 2012; Liebl et al., 2013). Analysis of microsatellite loci and methylation-sensitive amplification polymorphisms (MSAP) revealed that the Kenyan house sparrow populations have low genetic diversity but high epigenetic diversity (Liebl et al., 2013). There was a significant negative correlation between genetic and epigenetic diversity (Figure 4A) and a positive correlation between epigenetic diversity and the inbreeding coefficient, suggesting that DNA methylation may help to overcome genetic barriers typically associated with invasions such as bottlenecks and inbreeding. Schrey et al.(2012) compared both invasions genetically and epigenetically. The house sparrows from Kenya had less genetic diversity at multiple microsatellite loci but higher overall methylation when compared to their Floridian relatives. However, some restriction fragments were more methylated in the Floridian population (Figure 4B). The authors concluded that epigenetic variation may have compensated for low genetic variation during house sparrow invasions facilitating colonization and establishment in new environments.

To investigate how fast phenotypic changes appear after invasion of a new environment, Hu et al. (2019) colonized eight small Caribbean islands with brown anole lizard, Anolis sagrei, from a common source population. Four days after release they recaptured specimens from islands with high and low habitat quality and determined genome-wide DNA methylation. They found that a significant proportion of the recorded epigenetic variation was explained by habitat quality. Cytosines were differently methylated in genes involved in signal transduction, circadian rhythm and immune response. The study demonstrates that epigenetic signatures can be changed already in the first days after colonisation and that these changes differ between habitats. 
Liew et al. (2020) analysed DNA methylation patterns by whole genome bisulfite sequencing in two populations of the reef-building brain coral, Platygyra daedalea, from strikingly different environments. The Abu Dhabi population inside the Arabian-Persian Gulf had experienced large annual temperature and salinity differences (<19degC to $>35 \mathrm{degC} ; 40-46 \mathrm{psu})$ and several coral bleaching events in the past decades. The Fujairah population lives outside the Gulf in less stressful temperature and salinity regimes $(22-33$ degC, 36-39 psu) and was not affected by coral bleaching. The authors identified 1.42 million CpG dinucleotides that were consistently methylated in the $\sim 800 \mathrm{Mb}$ genome, corresponding to $3.2 \%$ of all CpGs. By comparing adults and their gametes and larvae, Liew et al. (2020) demonstrated that the DNA methylation patterns were determined by genotype, developmental stage and the parental environment (Figure 4C, D). Moreover, genome-wide $\mathrm{CpG}$ methylation was inherited from adults to their sperm and larvae as supported by crossing experiments, suggesting the involvement of transgenerational epigenetic inheritance in adaptation to local temperature and salinity regimes. The authors also found that genotype-independent changes of methylation levels in stress-related genes were strongly correlated with offspring survival under heat stress. These findings strongly support a role of DNA methylation and transgenerational epigenetic inheritance in adaptation of brain coral populations to different environments.

\section{Ecological implications of epigenetic phenotypes and ecotypes}

At first glance, epigenetic phenotypes and ecotypes seem to be of minor significance in animal ecology, because asexuals as the main benefiters are outnumbered by the sexually reproducing species. This is in contrast to bacteria, protists, fungi and plants, in which asexual reproduction is the rule or very common. However, asexual reproduction is also common in several sessile animal taxa including sponges, cnidarians, bryozoans and ascidians and some parasitic groups such as trematodes and cestodes. Moreover, widespread agricultural pests like aphids and key species of aquatic food chains like water fleas often reproduce by facultative parthenogenesis (Vrijenhoek, 1998; Schon et al., 2009).

Research with clonal animals like marbled crayfish has shown that epigenetic variation is independent from DNA sequence variation, but, of course, not independent from the information encoded in the DNA. In sexually reproducing animals, epigenetic variation is only semi-independent from genetic variation because the number of CpGs in the DNA, which significantly contribute to the epigenetic code, can vary among population members.

Theoretically, the environmental adaptation of the clonal animals described above and the establishment of epigenetic ecotypes could result from the repeated de novo production of identical, environmentally-induced phenotypes in each generation and/or the transgenerational inheritance and selection of adaptive epigenotypes. The example of the brain coral and recent reviews on the inheritance of epigenotypes (Jablonka, 2017; Anastasiadi et al., 2021; Feiner, 2021; Skinner \& Nilsson, 2021) suggest that transgenerational epigenetic inheritance can play an important role in long-term adaptation of genetically uniform populations to different environments, indeed. Final answering of this question requires cross-transplantation experiments and monitoring of epigenetic fingerprints for dozens of generations, which have not been done as yet.

Epigenetically-based phenotypic variation is assumed to play different roles in different biological contexts. In asexually reproducing species and lineages, the production of epigenetic phenotypes and epigenetic ecotypes from the same DNA sequence seems to be indispensable for adaptation to different environments. In small, genetically impoverished invasive groups, epigenetic variation is particularly important for the survival of the invasive generation and establishment in the new environment. In genetically diverse sexually reproducing species, epigenetic phenotypes may mainly serve to cope with rapid environmental changes. Epigeneticallybased phenotypic responses to new environmental cues affect many population members simultaneously in the first exposed generation, whereas adaptive genetic mutations and beneficial meiotic gene combinations first occur in single specimens and require many generations to become dominant in the population. Due to this reason, the use of epigenetic variation should be particularly advantageous for animals with long generation times. It should also be particularly useful to sessile species that cannot evade unfavourable conditions by migration. 
Combined with transgenerational epigenetic inheritance the production of epigenetic phenotypic variation would be a perfect means to cope with transient environmental stressors and environmental changes (Burggren, 2016). If the triggering conditions should disappear in the lifetime of the exposed generation or the subsequent generation, the epigenetic marks and related phenotypes could be reverted to the old state, but when the eliciting conditions should become permanent the epigenetic variants may persist and get selected resulting in the establishment of epigenetic ecotypes.

The generation of epigenetic phenotypes from the same genome and adaptation to new conditions by environmentally-induced epigenetic variation are supposedly the molecular mechanisms underlying the "general-purpose genotype" (Massicotte \& Angers, 2012) and the "genetic paradox of invasion" (Hawes et al., 2018).

\section{Evolutionary potential of epigenetic phenotypes and ecotypes}

The evolutionary potential of epigenetic signatures and related phenotypic traits is much dependent on whether they can be inherited across generations and genetically integrated in the long-term. Empirical research revealed that epigenetic marks and phenotypes can be inherited across generations, indeed. However, most studies in animals were run for 1-3 generations only (Casas \& Vavouri, 2020) and new studies over many more generations are necessary to obtain a clear picture. Experiments have also shown that the extent of epigenetic inheritance depends very much on trait and condition. Modelling revealed that long-term effects of epimutations depend crucially on their stability and fitness effects relative to genetic mutations (Kronholm \& Collins, 2016). Sperm seems to be particularly effective in transmitting epigenetic information to the next generation via conservation of DNA methylation, histone modifications and ncRNAs, as exemplified in taxonomically distant corals and rat (Liew et al., 2020; Beck et al., 2021).

In a well adapted population living in a constant environment it makes little sense to inherit epigenetic variants over many generations because it may incur costs but provide no advantage. However, if the environment changes from one stable condition to another stable condition, then new and better suited epigenetic variants may be selected and stably inherited. Interestingly, the nematode and epigenetics model Caenorhabditis elegans was shown to possess a timing mechanism based on ncRNAs that controls the duration of transgenerational epigenetic inheritance (Houri-Ze'evi \& Rechavi, 2017), which may help to maintain or reset an epigenetic phenotype.

In sexually reproducing species, beneficial epigenetic phenotypes can be fixed on the long term by genetic assimilation, a process by which a phenotype originally produced in response to environmental signals is later taken over by the genotype via selection on random genetic mutations with similar phenotypic effects (Waddington, 1953; Ehrenreich \& Pfennig, 2016). An alternative, more directional mechanism, which would also be applicable to asexually reproducing species, is the facilitated mutation of epimutations with phenotypic effects to corresponding genetic mutations. For example, methylated CpGs mutate into TpGs in a 10-50 fold higher probability than unmethylated CpGs (Lutsenko \& Bhagwat, 1999). The biological significance of such mutations is well documented for bacteria, Methylated cytosines were identified as hot spots for cytosine-to-thymine mutations that changed crucial fitness traits like antibiotics susceptibility (Gosh et al., 2020). In animals, phenotypically relevant $\mathrm{mCpG}$ to $\mathrm{TpG}$ transitions can occur in relatively short periods of time as demonstrated for domesticated European seabass, Dicentrarchus labrax. Several methylated CpGs that were established from unmethylated CpGs in the second generation of domestication in response to the new culture conditions appeared as TpGs after 25 generations (Anastasiadi \& Pifferer, 2019). This way, a principally reversible, epigenetically determined phenotype could become a permanent, genetically encoded phenotype. The long-term genetic integration of epigenetic phenotypes may also lead to the transformation of epigenetic ecotypes into classical, genetically diverse ecotypes.

According to traditional belief, clonal lineages are dead ends of evolution because of the absence of genetic recombination, the most effective mechanism to create new phenotypes.

However, the bdelloid rotifers that lived without sex for 40 million years (Mark Welch \& Meselson, 2000) yielded 4 families, 18 genera, and 360 species. A similar diversity evolved in some obligate parthenogenetic 
freshwater ostracod groups, which exist for more than 100 million years without sex (Butlin et al., 1998). The diversity of such evolutionarily successful

asexuals is usually explained with the temporal appearance of sexually reproducing individuals, the separate origin of clones from different sexual ancestors, and hybridization between asexual females and males from related species, but there is no proof for these hypotheses. An alternative explanation might be speciation via the following sequence: environmental induction of different epigenetic phenotypes - epigenetic ecotypes - genetic ecotypes - new species (Figure 5).

\section{Conclusions}

(1) Recent investigations of clonal animals with advanced genetic and epigenetic whole genome approaches revealed that different phenotypes can be produced from the same DNA sequence via gene expression change through environment-responsive epigenetic mechanisms. This mode of phenotype production is the basis of epigenetic ecotypes, the general-purpose genotype and the genetic paradox of invasion.

(2) The generation of epigenetic phenotypes is particularly important for the persistence of asexual and genetically impoverished populations but may also help genetically diverse populations to adapt to rapidly changing environments. Aside of asexual reproducers, sessile animals that cannot evade unfavourable environmental conditions by migration and species with long generation times seem to particularly profit from this overlooked type of phenotype generation.

(3) There is sound evidence that asexuals can establish stable epigenetic ecotypes in the virtual absence of genetic variation. However, since epigenetic fingerprints in differently adapted populations were studied for only a few consecutive years, it remains to be investigated how exactly epigenetic ecotypes are maintained and what role transgenerational epigenetic inheritance plays in this context.

(4) It remains to be investigated how frequent epigenetic ecotypes are in nature, how much epigenetic mechanisms contribute to ecological adaptation in sexually reproducing populations, how and under what conditions epigenetic phenotypes and epigenetic ecotypes are maintained over decades, and whether epigenetic ecotypes can evolve into classical ecotypes.

(5) The consideration of environmentally-induced epigenetic phenotypic variation as a potential substrate for selection in addition to genetic phenotypic variation may broaden our general understanding of ecological adaptation, the response of organisms to rapid environmental change, biological invasions, and the evolution of asexual organisms.

\section{CONFLICT OF INTEREST}

The author declares no conflict of interest.

\section{DATA AVAILABILITY STATEMENT}

Not applicable.

ORCID

Gunter Vogt https://orcid.org/0000-0002-5632-2477

\section{References}

Allis, C. D., \& Jenuwein, T. (2016). The molecular hallmarks of epigenetic control. Nature Reviews Genetics , 17, 487-500.

Anastasiadi, D., \& Piferrer, F. (2019). Epimutations in developmental genes underlie the onset of domestication in farmed European Sea Bass. Molecular Biology and Evolution , 36 , 2252-2264.

Anastasiadi, D., Venney, C. J., Bernatchez, L., \& Wellenreuther, M. (2021). Epigenetic inheritance and reproductive mode in plants and animals. Trends in Ecology and Evolution , 36 , 1124-1140. 
Andriantsoa, R., Tonges, S., Panteleit, J., Theissinger, K., Coutinho Carneiro, V., Rasamy, J., \& Lyko, F. (2019). Ecological plasticity and commercial impact of invasive marbled crayfish populations in Madagascar. BMC Ecology , 19 , 8 .

Angers, B., Perez, M., Menicucci, T., \& Leung, C. (2020). Sources of epigenetic variation and their applications in natural populations.Evolutionary Applications , 13 , 1262-1278.

Ballouz, S., Pena, M. T., Knight, F. M., Adams, L. B., \& Gillis, J. A. (2019). The transcriptional legacy of developmental stochasticity.bioRxiv , preprint. https://doi.org/10.1101/2019.12.11.873265

Beck, D., Ben Maamar, M., \& Skinner, M. K. (2021). Integration of sperm ncRNA-directed DNA methylation and DNA methylation-directed histone retention in epigenetic transgenerational inheritance. Epigenetics $\mathcal{G}^{3}$ Chromatin, 14,6 .

Burggren, W. (2016). Epigenetic inheritance and its role in evolutionary biology: re-evaluation and new perspectives. Biology, 5, 24.

Butlin, R., Schon, I., \& Martens, K. (1998). Asexual reproduction in nonmarine ostracods. Heredity, 81 , 473-480.

Casas, E., \& Vavouri, T. (2020). Mechanisms of epigenetic inheritance of variable traits through the germline. Reproduction ,159, R251-R263.

Coutinho Carneiro, V., \& Lyko, F. (2020). Rapid epigenetic adaptation in animals and its role in invasiveness. Integrative and Comparative Biology , 60 , 267-274.

Danisman, S. (2016). TCP transcription factors at the interface between environmental challenges and the plant's growth responses. Frontiers in Plant Science, 7 , 1930.

Du, Q., Luu, P.-L., Stirzaker, C., \& Clark, S. J. (2015). Methyl-CpG-binding domain proteins: readers of the epigenome.Epigenomics , 7 , 1051-1073.

Dybdahl, M. F., \& Drown, D. M. (2011). The absence of genotypic diversity in a successful parthenogenetic invader. Biological Invasions , $13,1663-1672$.

Ehrenreich, I. M., \& Pfennig, D. W. (2016). Genetic assimilation: a review of its potential proximate causes and evolutionary consequences.Annals of Botany , 117, 769-779.

Estoup, A., Ravigne, V., Hufbauer, R., Vitalis, R., Gautier, M., \& Facon, B. (2016). Is there a genetic paradox of biological invasion? Annual Review of Ecology, Evolution and Systematics , 47 , 51-72.

Feinberg, A. P., \& Irizarry, R. A. (2010). Stochastic epigenetic variation as a driving force of development, evolutionary adaptation, and disease. Proceedings of the National Academy of Sciences of the United States of America, Supplement 1, 1757-1764.

Feiner, N., Radersma, R., Vasquez, L., Ringner, M., Nystedt, B., Raine, A., Tobi, E. W., Heijmans, B. T., \& Uller, T. (2021). Environmentally-induced DNA methylation is inherited across generations in an aquatic keystone species (Daphnia magna ). bioRxiv , preprint. https://doi.org/10.1101/2021.12.05.471257

Foquet, B., Castellanos, A. A., \& Song, H. (2021). Comparative analysis of phenotypic plasticity sheds light on the evolution and molecular underpinnings of locust phase polyphenism. Scientific Reports ,11, 11925.

Gatzmann, F., Falckenhayn, C., Gutekunst, J., Hanna, K., Raddatz, G., Coutinho Carneiro, V., \& Lyko, F. (2018). The methylome of the marbled crayfish links gene body methylation to stable expression of poorly accessible genes. Epigenetics \& Chromatin , $11,57$.

Ghosh, D., Veeraraghavan, B., Elangovan, R., \& Vivekanandan, P. (2020). Antibiotic resistance and epigenetics: more to it than meets the eye.Antimicrobial Agents and Chemotherapy, 64, e02225-19. 
Guillette, L. J. Jr., Parrott, B. B., Nilsson, E., Haque, M. M., \& Skinner, M. K. (2016). Epigenetic programming alterations in alligators from environmentally contaminated lakes. General and Comparative Endocrinology , 238, 4-12.

Gutekunst, J., Andriantsoa, R., Falckenhayn, C., Hanna, K., Stein, W., Rasamy, J., \& Lyko, F. (2018). Clonal genome evolution and rapid invasive spread of the marbled crayfish. Nature Ecology and Evolution, 2, 567-573.

Gutekunst, J., Maiakovska, O., Hanna, K., Provataris, P., Horn, H., Wolf, S., Skelton, C. E., Dorn, N. J., \& Lyko, F. (2021). Phylogeographic reconstruction of the marbled crayfish origin. Communications Biology , $4,1096$.

Hawes, N. A., Fidler, A. E., Dunphy, B. J., Smith, K. F., Tremblay, L. A., \& Pochon, X. (2018). Understanding the role of DNA methylation in successful biological invasions: a review. Biological Invasions ,20 , 2285-2300.

Houri-Ze'evi, L., \& Rechavi, O. (2017). A matter of time: small RNAs regulate the duration of epigenetic inheritance. Trends in Genetics , 33 , 46-57.

Hu, J., Askary, A. M., Thurman, T. J., Spiller, D. A., Palmer, T. M., Pringle, R. M., \& Barrett, R. (2019). The epigenetic signature of colonizing new environments in anolis lizards. Molecular Biology and Evolution , $36,2165-2170$.

Huang, Q., Ma, C., Chen, L., Luo, D., Chen, R., \& Liang, F. (2018). Mechanistic insights into the interaction between transcription factors and epigenetic modifications and the contribution to the development of obesity. Frontiers in Endocrinology , $9,370$.

Jablonka, E. (2017). The evolutionary implications of epigenetic inheritance. Interface Focus , 7 , 20160135.

Jaenisch, R., \& Bird, A. (2003). Epigenetic regulation of gene expression: how the genome integrates intrinsic and environmental signals. Nature Genetics , 33 , 245-254.

Kernaleguen, M., Daviaud, C., Shen, Y., Bonnet, E., Renault, V., Deleuze, J.-F., Mauger, F., \& Tost, J. (2018). Whole-genome bisulfite sequencing for the analysis of genome-wide DNA methylation and hydroxymethylation patterns at single-nucleotide resolution. Methods in Molecular Biology , 1767 , 311-349.

Kribelbauer, J. F., Lu, C.-J., Rohs, R., Mann, R. S., \& Bussemaker, H. J. (2020). Toward a mechanistic understanding of DNA methylation readout by transcription factors. Journal of Molecular Biology , 432 , $1801-1815$.

Kronholm, I., \& Collins, S. (2016). Epigenetic mutations can both help and hinder adaptive evolution. Molecular Ecology , 25 , 1856-1868.

Kvist, J., Goncalves Athanasio, C., Pfrender, M. E., Brown, J. B., Colbourne, J. K., \& Mirbahai, L. (2020). A comprehensive epigenomic analysis of phenotypically distinguishable, genetically identical female and male Daphnia pulex . BMC Genomics , 21, 7.

Legrand, C., Andriantsoa, A., Lichter, P., \& Lyko, F. (2021). Time-resolved, integrated analysis of clonal genome evolution in parthenogenetic animals and in cancer. bioRxiv, preprint. https://doi.org/10.1101/2021.10.08.463633

Lennartsson, A., \& Ekwall, K. (2009). Histone modification patterns and epigenetic codes. Biochimica Biophysica Acta , 1790 , 863-868.

Leung, C., Breton, S., \& Angers, B. (2016). Facing environmental predictability with different sources of epigenetic variation.Ecology and Evolution , 6 , 5234-5245.

Liebl, A. L., Schrey, A. W., Richards, C. L., \& Martin, L. B. (2013). Patterns of DNA methylation throughout a range expansion of an introduced songbird. Integrative and Comparative Biology ,53 , 351-358. 
Liew, Y. J., Zoccola, D., Li, Y., Tambutte, E., Venn, A. A., Michell, C. T., Cui, G., Deutekom, E. S., Kaandorp, J. A., Voolstra, C. R., Foret, S., Allemand, D., Tambutte, S., \& Aranda, M. (2018). Epigenomeassociated phenotypic acclimatization to ocean acidification in a reef-building coral. Science Advances , 4 , eaar8028.

Liew, Y. J., Howells, E. J., Wang, X., Michell, C. T., Burt, J. A., Idaghdour, Y., \& Aranda, M. (2020). Intergenerational epigenetic inheritance in reef-building corals. Nature Climate Change ,10 , 254-259.

Lowry, D. B. (2012). Ecotypes and the controversy over stages in the formation of new species. Biological Journal of the Linnean Society, 106 , 241-257.

Lutsenko, E., \& Bhagwat, A. S. (1999). Principal causes of hot spots for cytosine to thymine mutations at sites of cytosine methylation in growing cells. A model, its experimental support and implications. Mutation Research , 437, 11-20.

Lyko, F. (2018). The DNA methyltransferase family: a versatile toolkit for epigenetic regulation. Nature Reviews Genetics , 19, 81-92.

Mackay-Smith, A., Dornon, M. K., Lucier, R., Okimoto, A., Mendonca de Sousa, F., Rodriguero, M., Confalonieri, V., Lanteri, A. A., \& Sequeira, A. S. (2021). Host-specific gene expression as a tool for introduction success in Naupactus parthenogenetic weevils.PLoS ONE , 16 , e0248202.

Maiakovska, O., Andriantsoa, R., Tonges, S., Legrand, C., Gutekunst, J., Hanna, K., Parvulescu, L., Novitsky, R., Weiperth, A., Sciberras, A., Deidun, A., Ercoli, F., Kouba, A., \& Lyko, F. (2021). Genome analysis of the monoclonal marbled crayfish reveals genetic separation over a short evolutionary timescale. Communications Biology , 4, 74 .

Malec, P., Weber, J., Bohmer, R., Fiebig, M., Meinert, D., Rein, C., Reinisch, R., Henrich, M., Polyvas, V., Pollmann, M., von Berg, L., Konig, C., \& Steidle, J. L. M. (2021). The emergence of ecotypes in a parasitoid wasp: a case of incipient sympatric speciation in Hymenoptera? BMC Ecology and Evolution , 21, 204.

Mark Welch, D. B., \& Meselson, M. (2000). Evidence for the evolution of bdelloid rotifers without sexual reproduction or genetic exchange.Science, 288, 1211-1215.

Marmorstein, R., \& Zhou, M.-M. (2014). Writers and readers of histone acetylation: structure, mechanism, and inhibition. Cold Spring Harbor Perspectives in Biology , 6 , a018762.

Martin, P., Dorn, N. J., Kawai, T., van der Heiden, C., \& Scholtz, G. (2010). The enigmatic Marmorkrebs (marbled crayfish) is the parthenogenetic form of Procambarus fallax (Hagen, 1870). Contributions to Zoology , $79,107-118$.

Martin, P., Thonagel, S., \& Scholtz, G. (2016). The parthenogenetic Marmorkrebs (Malacostraca: Decapoda: Cambaridae) is a triploid organism. Journal of Zoological Systematics and Evolutionary Research ,54, 13-21.

Massicotte, R., \& Angers, B. (2012). General-purpose genotype or how epigenetics extend the flexibility of a genotype. Genetics Research International , 2012, 317175.

Morgan, M. A. J., \& Shilatifard, A. (2020). Reevaluating the roles of histone-modifying enzymes and their associated chromatin modifications in transcriptional regulation. Nature Genetics , 52 , 1271-1281.

Moutinho, C., \& Esteller, M. (2017). MicroRNAs and epigenetics.Advances in Cancer Research , 135 , $189-220$.

Norouzitallab, P., Baruah, K., Biswas, P., Vanrompay, D., \& Bossier, P. (2016). Probing the phenomenon of trained immunity in invertebrates during a transgenerational study, using brine shrimp Artemia as a model system. Scientific Reports , 6 , 21166.

Peck, J. R., Yearsley, J. M., \& Waxman, D. (1998). Explaining the geographic distributions of sexual and asexual populations.Nature, $391,889-892$. 
Ravichandran, M., Jurkowska, R. Z., \& Jurkowski, T. P. (2018). Target specificity of mammalian DNA methylation and demethylation machinery.Organic \&S Biomolecular Chemistry , 16 , 1419.

Sax, D. F., \& Brown, J. H. (2000). The paradox of invasion. Global Ecology and Biogeography , 9 , 363-371.

Schon, I., Martens, K., \& Dijk, P., Eds. (2009). Lost sex: The evolutionary biology of parthenogenesis . Springer.

Schrey, A. W., Coon, C. A. C., Grispo, M. T., Awad, M., Imboma, T., McCoy, E. D., Mushinsky, H. R,, Richards, C. L., \& Martin, L. B. (2012). Epigenetic variation may compensate for decreased genetic variation with introductions: a case study using house sparrows (Passer domesticus) on two continents. Genetics Research International, 2012, 979751.

Simpson, S. J., Sword, G. A., \& Lo, N. (2011). Polyphenism in insects.Current Biology , 21 , R738-R749.

Skinner, M. K. (2014). Endocrine disruptor induction of epigenetic transgenerational inheritance of disease. Molecular and Cellular Endocrinology , 398 , 4-12.

Skinner, M. K., \& Nilsson, E. E. (2021). Role of environmentally induced epigenetic transgenerational inheritance in evolutionary biology: unified evolution theory. Environmental Epigenetics ,7 , 1-12.

Thorson, J. L. M., Smithson, M., Beck, D., Sadler-Riggleman, I., Nilsson, E., Dybdahl, M., \& Skinner, M. K. (2017). Epigenetics and adaptive phenotypic variation between habitats in an asexual snail.Scientific Reports , 7, 14139.

Thorson, J .L. M., Smithson, M., Sadler-Riggleman, I., Beck, D., Dybdahl, M., \& Skinner, M. K. (2019). Regional epigenetic variation in asexual snail populations among urban and rural lakes.Environmental Epigenetic , 5, dvz020.

Tonges, S., Venkatesh, G., Andriantsoa, R., Hanna, K., Gatzmann, F., Raddatz, F., Coutinho Carneiro, V., \& Lyko, F. (2021a). Location-dependent DNA methylation signatures in a clonal invasive crayfish. Frontiers in Cell and Developmental Biology, 9 , 794506.

Tonges, S., Masagounder, K., Lenich, F., Gutekunst, J., Tonges, M., Lohbeck, J., Miller, A. K., Bohl, F., \& Lyko, F. (2021b). Evaluating invasive marbled crayfish as a potential livestock for sustainable aquaculture. Frontiers in Ecology and Evolution , 9 , 651981.

Van der Graaf, A., Wardenaar, R., Neumann, D. A., Taudt, A., Shaw, R. G., Jansen, R. C., Schmitz, R. J., Colome-Tatche, M., \& Johannes, F. (2015). Rate, spectrum, and evolutionary dynamics of spontaneous epimutations. Proceedings of the National Academy of Sciences of the United States of America, 112, 6676-6681.

Van Dijk, E. L., Jaszczyszyn, Y., Naquin, D., \& Thermes, C. (2018). The third revolution in sequencing technology. Trends in Genetics ,34, 666-681.

Van Dongen, J., Gordon, S. D., McRae, A. F., Odintsova, V. V., Mbarek, H., Breeze, C. E., Sugden, K., Lundgren, S., Castillo-Fernandez, J. E., Hannon, E., Moffitt, T. E., Hagenbeek, F. A., van Beijsterveldt, C. E. M., Hottenga, J. J., Tsai, P.-C., et al. (2021). Identical twins carry a persistent epigenetic signature of early genome programming. Nature Communications , 12 , 5618.

Verhoeven, K. J. F., \& Preite, V. (2014). Epigenetic variation in asexually reproducing organisms. Evolution , 68, 644-655.

Vickaryous, M. K., \& Hall, B. K. (2006). Human cell type diversity, evolution, development, and classification with special reference to cells derived from the neural crest. Biological Reviews ,81 , 425-455.

Vogt, G. (2017). Facilitation of environmental adaptation and evolution by epigenetic phenotype variation: insights from clonal, invasive, polyploid, and domesticated animals. Environmental Epigenetics ,3 , dvx002. 
Vogt, G. (2019). Functional cytology of the hepatopancreas of decapod crustaceans. Journal of Morphology , $280,1405-1444$.

Vogt, G. (2020a). Disentangling the environmentally induced and stochastic developmental components of phenotypic variation. In H. Levine, M. K. Jolly, P. Kulkarni, \& V. Nanjundiah (Eds.), Phenotypic switching: Implications in biology and medicine (pp. 207-251). Academic Press.

Vogt, G. (2020b). Biology, ecology, evolution, systematics and utilization of the parthenogenetic marbled crayfish, Procambarus virginalis . In F. B. Ribeiro (Ed.), Crayfish: Evolution, habitat and conservation strategies (pp. 137-227). Nova Science Publishers.

Vogt, G. (2021). Epigenetic variation in animal populations: sources, extent, phenotypic implications, and ecological and evolutionary relevance. Journal of Biosciences , 46 , 24.

Vogt, G. (2022). Studying phenotypic variation and DNA methylation across development, ecology and evolution in the clonal marbled crayfish: a paradigm for investigating epigenotype-phenotype relationships in macro-invertebrates. The Science of Nature ,109, 16.

Vogt, G., Huber, M., Thiemann, M., van den Boogaart, G., Schmitz, O. J., \& Schubart, C. D. (2008). Production of different phenotypes from the same genotype in the same environment by developmental variation. Journal of Experimental Biology , 211 , 510-523.

Vogt, G., Falckenhayn, C., Schrimpf, A., Schmid, K., Hanna, K., Panteleit, J., Helm, M., Schulz, R., \& Lyko, F. (2015). The marbled crayfish as a paradigm for saltational speciation by autopolyploidy and parthenogenesis in animals. Biology Open , 4 , 1583-1594.

Vogt, G., Lukhaup, C., Pfeiffer, M., Dorn, N. J., Williams, B. W., Schulz, R., \& Schrimpf, A. (2018). Morphological and genetic characterization of the marbled crayfish, including a determination key.Zootaxa, 4524, 329-350.

Vogt, G., Dorn, N. J., Pfeiffer, M., Lukhaup, C., Williams, B. W., Schulz, R., \& Schrimpf, A. (2019). The dimension of biological change caused by autotriploidy: a meta-analysis with the triploidProcambarus virginalis and its diploid parent Procambarus fallax. Zoologischer Anzeiger , 281 , 53-67.

Voigt, S., \& Kost, L. (2021). Differences in temperature-sensitive expression of PcG regulated genes among natural populations ofDrosophila melanogaster. G3 Genes Genomes Genetics ,11, jkab237.

Vrijenhoek, R. C. (1998). Animal clones and diversity: are natural clones generalists or specialists? BioScience , 48, 617-628.

Waddington, C. H. (1953). Genetic assimilation of an acquired character.Evolution, 7 , 118-126.

Wu, H., \& Zhang, Y. (2017). TET-mediated active DNA demethylation: mechanism, function and beyond. Nature Reviews Genetics ,18 , 517-534.

Zhu, T., Brown, A. P., \& Ji, H. (2020). The emerging role of ten-eleven translocation 1 in epigenetic responses to environmental exposures.Epigenetics Insights , 13 , 1-9. 

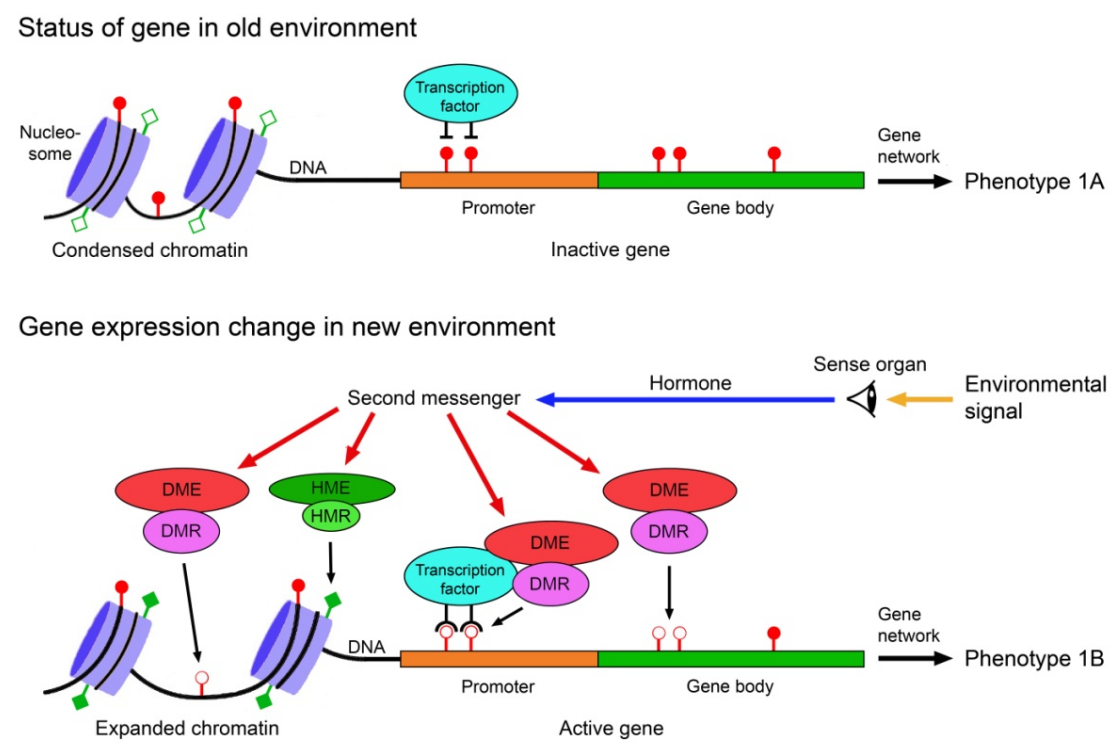

Figure. 1 Scheme of environmentally-induced changes of gene and phenotype expression via epigenetic mechanisms. Strong environmental cues can trigger gene expression change via signal transmission by hormones and second messengers and the concerted action of protein complexes including environment-sensitive DNA methylation modifying enzymes (DME) and histone modifying enzymes (HME). DNA methylation readers (DMR), histone modification readers (HMR) and transcription factors recruit the DMEs and HMEs to specific sites in the chromatin and DNA. Histone modifications like acetylation (filled squares) and deacetylation (open squares) change chromatin structure and access to the DNA, and methylation (filled circles) and demethylation (open circles) of $\mathrm{CpG}$ dinucleotides in the DNA modify gene expression, finally resulting in different variants of a phenotypic trait. 


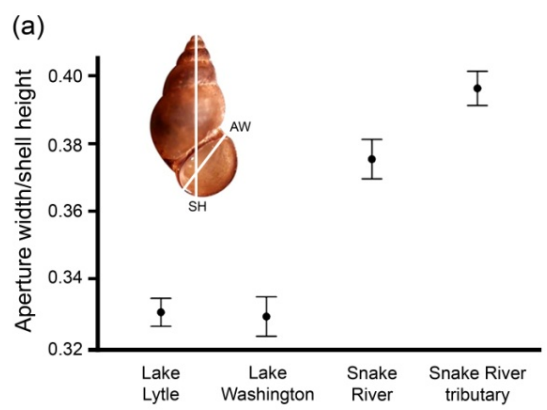

(c)

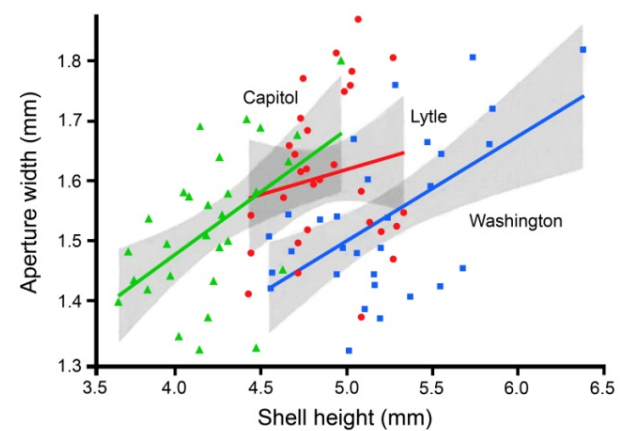

(b)
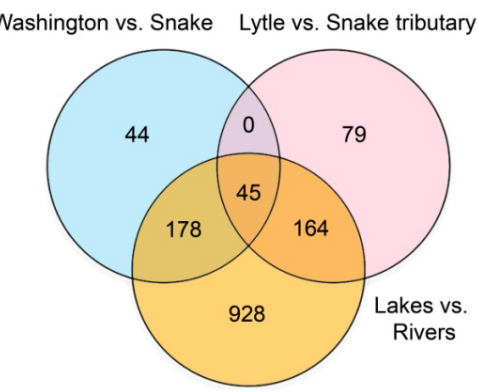

(d) Lytle vs. Capitol Lytle vs. Washington

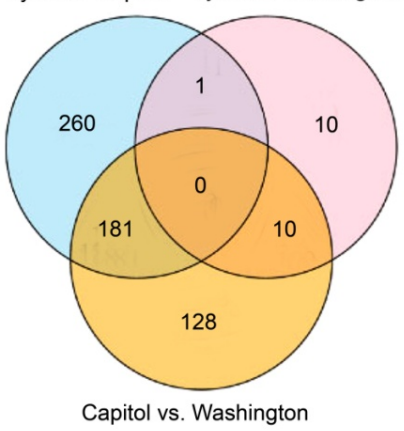

Figure 2 Environment-dependent variation of shell shape and DNA methylation in populations of monoclonal freshwater snailPotamopyrgus antipodarum. (a) Differences in shell shape between lentic and lotic habitats. AW, aperture width; SH, shell height (based on Thorson et al., 2017). (b) Venn diagram showing considerable numbers of differently methylated regions (DMRs) in lake versus river comparisons (based on Thorson et al., 2017). (c) Allometric shell growth differences between rural Lake Lytle, urban Capitol Lake and polluted Lake Washington. Shaded areas: 95\% confidence intervals (based on Thorson et al., 2019). (d) Comparison of DMRs between the same lakes, showing relatively small overlap between rural and polluted lake (based on Thorson et al., 2019). 
(a)

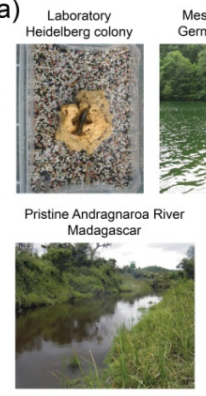

(c)

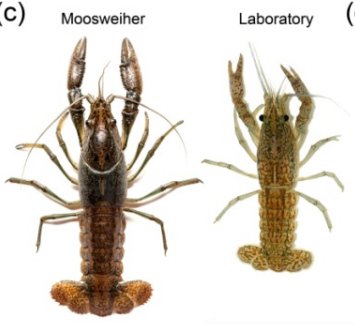

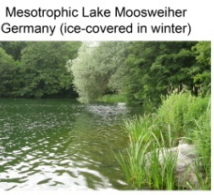
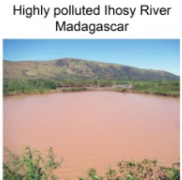

Acidic Lake Murner See
Germany (pH 3.9)

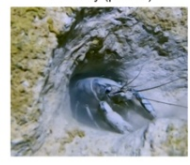

Thermal rice field
Madagascar $\left(37^{\circ} \mathrm{C}\right)$

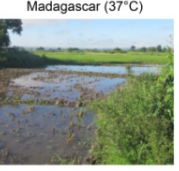

(b)

d) Moosweiner

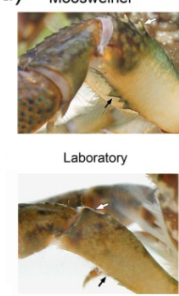

(e)

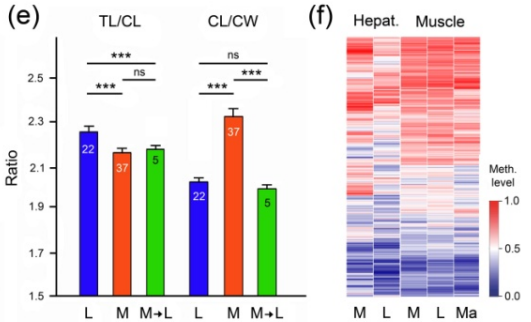

(g)

g) Singliser See Laboratory

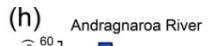

(i) Malagasy rivers German lakes
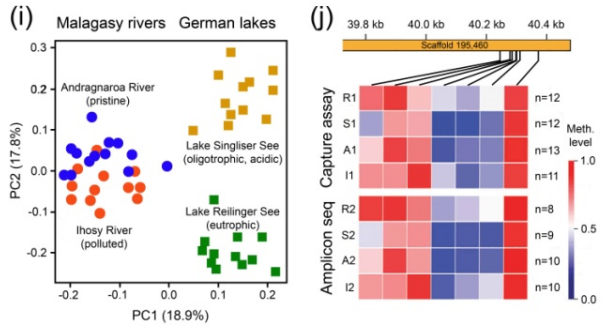

Figure 3 Genetic, epigenetic and phenotypic differences between differently adapted populations of marbled crayfish, Procambarus virginalis . (a) Examples of strikingly different marbled crayfish habitats (from Vogt et al., 2018; Andriantsoa et al., 2019; Tönges et al., 2021a, b). (b) Genetic differences between representatives from different European populations as determined by whole-genome sequencing. A descendant of the oldest known marbled crayfish laboratory population was used as a reference. G, Germany (Maiakovska et al., 2021). (c) Maximum body sizes of laboratory raised and wild specimens from Lake Moosweiher (Germany), showing $30 \%$ bigger growth in the lake (from Vogt et al., 2018). (d) Chelipeds of specimens from the laboratory and Lake Moosweiher, showing bigger and sharper spines (arrows) in the wild specimen (from Vogt et al., 2018). (e) Body proportions of marbled crayfish from the laboratory (L) and Lake Moosweiher (M) showing significant differences in total length/carapace length ratio (TL/CL) and carapace length/carapace width ratio $(\mathrm{CL} / \mathrm{CW})$. The adult offspring of a female that was transferred from the lake to the laboratory (M-L) and reproduced there had a TL/CL ratio similar to the wild population but a CL/CW ratio similar to the laboratory population. Figures in columns give numbers of specimens investigated; ${ }^{* * *}$ significantly different $(P<0.001)$; ns, not significantly different (based on Vogt, 2021). (f) Comparative analysis of 697 variably methylated genes in the hepatopancreas and abdominal musculature of specimens from the laboratory, Lake Moosweiher and a rice field in Moramanga, Madagascar (Ma). The heatmap shows differences in methylation patterns between individuals, particularly in the hepatopancreas (based on Tonges et al., 2021b). (g) Principal component analysis based on the average methylation of 361 variably methylated genes, showing clear separation of individuals from acidic Lake Singliser See and the laboratory (based on Tonges et al., 2021b). (h) Significant differences in population structure between pristine and montane Andragnaroa River and heavily polluted Ihosy River in Madagascar. $P<0.05$ (based on Andriantsoa et al., 2019). (i) Principal component analysis of methylation of 122 genes separating four populations from rivers and lakes in Germany and Madagascar (based on Tonges et al., 2021b). (j) Persistent DNA methylation fingerprints of populations 
from Andragnaroa River (A), Ihosy River (I), Lake Reilinger See (R) and Lake Singliser See (S) in consecutive years (1 and 2), exemplified for a small genic region of the hepatopancreatic DNA. The samples were collected at intervals of 12-21 months and analysed with two different methods (based on Tonges et al., 2021b).

(a)
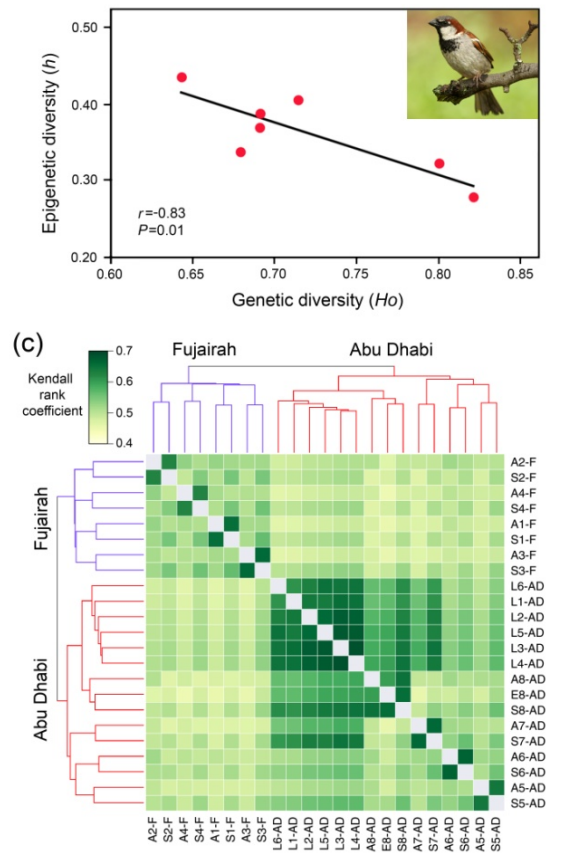

(b)

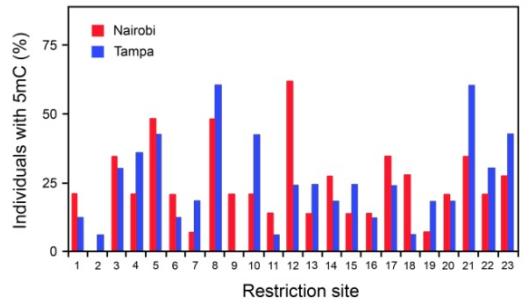

(d)

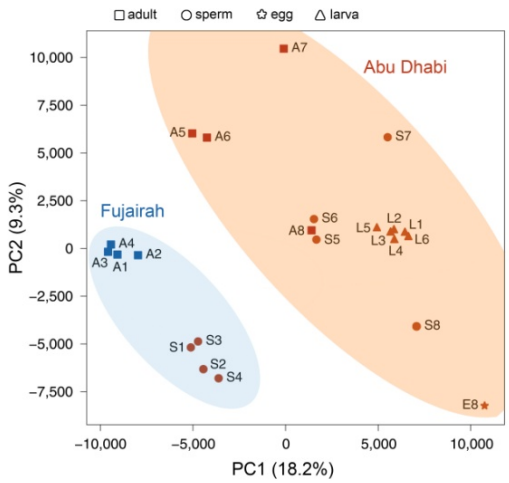

Figure 4 Site-specific epigenetic variation in differently adapted bird and coral populations. (a) Invasive house sparrow, Passer domesticus, from seven Kenyan cities showing negative correlation of genetic (microsatellites) and epigenetic (MSAP) variation. $h$, haplotype diversity; Ho, heterozygosity (based on Liebl et al., 2013). (b) Comparison of house sparrow invasions of different age in Nairobi, Kenya ( $65 \mathrm{yr}, \mathrm{n}=14$ ) and Tampa, Florida ( $150 \mathrm{yr}, \mathrm{n}=16$ ). The populations differ markedly in methylated CpGs of 23 restriction sites (based on Schrey et al., 2012). (c) Comparison of populations of brain coral, Platygyra daedalea, from rather stable environment in Fujairah (F, specimens 1-4) and thermally and salinely fluctuating environment in Abu Dhabi (AD, specimens 5-8). Analysed were adults (A), their sperm (S) and eggs (E), and larval offspring from reciprocal crosses between E7 and S8 (L1-L3) and S7 and E8 (L4-L6). Pair-wise correlation of methylation data revealed grouping of samples by environmental origin. Moreover, gametes and larvae cluster best with their parents suggesting inheritance of methylation patterns (based on Liew et al., 2020). (d) Principal component analysis of DNA methylation demonstrating separation of samples due to environment and developmental stage (based on Liew et al., 2020). 


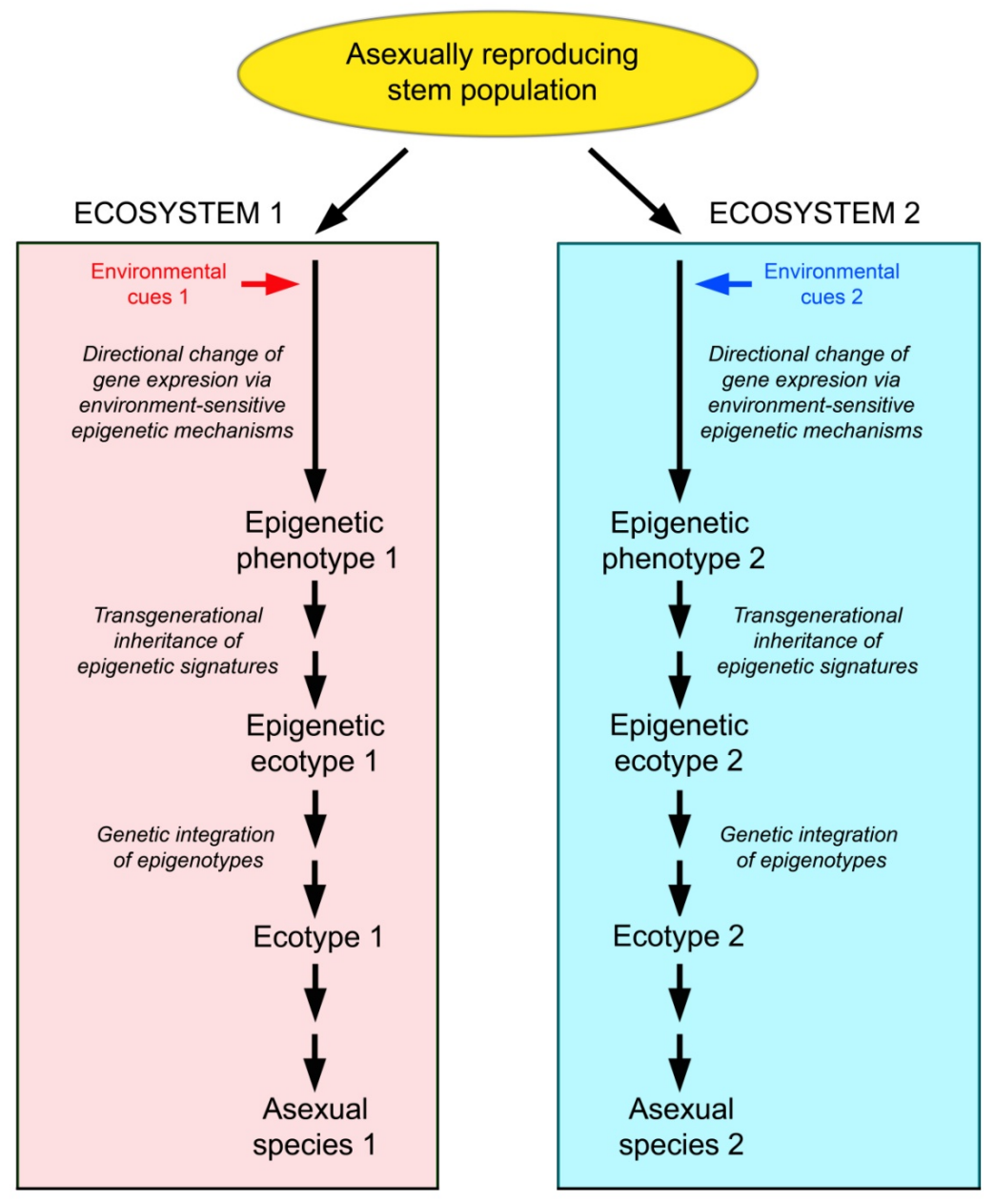

Figure 5 Scheme of the establishment of epigenetic ecotypes and their possible evolutionary potential. Epigenetic ecotypes can arise from an asexually reproducing, genetically identical source population by invasion of different ecosystems, the generation of habitat-specific phenotypes by environmentally induced epigenetic mechanisms, and the transgenerational inheritance and selection of these epigenetic phenotypes. Under favourable conditions, the epigenotypes may be genetically integrated in the long term and the epigenetic ecotypes may end up in classical, genetically diverse ecotypes and finally even in different species. 